\title{
Anti-angiogenic effect of the total flavonoids in Scutellaria barbata D. Don
}

\author{
Zhi-Jun Dai ${ }^{1 *}$, Wang-Feng Lu ${ }^{1,2+}$, Jie Gao ${ }^{3+}$, Hua-Feng Kang ${ }^{1}$, Yu-Guang Ma', Shu-Qun Zhang ${ }^{1}$, Yan Diao , \\ Shuai Lin ${ }^{1}, X^{i-J i n g ~ W a n g}{ }^{1 *}$ and Wen-Ying Wu ${ }^{4^{*}}$
}

\begin{abstract}
Background: Angiogenesis is closely related to the growth, invasion and metastasis of tumors, also considered as the key target of anticancer therapy. Scutellaria barbata D. Don (S. barbata), a traditional Chinese medicine, is being used to treat various diseases, including cancer. However, the antitumor molecular mechanism of S. barbata was still unclear. This study aimed to investigate the inhibitory effects of the total flavones in S. barbata (TF-SB) on angiogenesis.

Methods: Human umbilical vein endothelial cells (HUVECS) were treated with various concentrations of TF-SB. Cell viability was examined using the MTT assay. The scratch assay was used to detect the migration of HUVECs after treatment with TF-SB. The ability of HUVECs to form network structures in vitro was demonstrated using the tube formation assay. The chick embryo chorioallantoic membrane assay was performed to detect the in vivo antiangiogenic effect. The expression of VEGF was measured by the enzyme-linked immunosorbent.
\end{abstract}

Results: Results showed that TF-SB inhibited the proliferation and migration of HUVECs in a dose- dependent manner. Simultaneously, TF-SB significantly suppressed HUVEC angiogenesis in vitro and in vivo. Furthermore, VEGF was downregulated in both HUVECS and MHCC97-H cells after TF-SB treatment.

Conclusion: TF-SB could suppress the process of angiogenesis in vitro and in vivo. TF-SB potentially suppresses angiogenesis in HUVECs by regulating VEGF. These findings suggested that TF-SB may serve as a potent antiangiogenic agent.

Keywords: Scutellaria Barbata, Angiogenesis, Hepatocellular Carcinoma, Human Umbilical Vein Endothelial Cells

\section{Background}

The incidence of hepatocellular carcinoma (HCC) in worldwide is on the rise. According to global statistics, approximately 7 million of people die each year from liver cancer which includes HCC [1]. The selection of HCC treatment depends on the tumor heterogeneity, biological behavior, and liver function [2]. Chemotherapy is one of the main methods for the treatment of HCC because it can completely destroy cancer cells. However, chemotherapy may cause severe simultaneous side-effects and often lead to multidrug resistance [3]. Therefore,

\footnotetext{
*Correspondence: dzj0911@126.com; wangxijing@21cn.com; wwy0413@126. com

${ }^{\dagger}$ Equal contributors

'Department of Oncology, the Second Affiliated Hospital of Xi'an Jiaotong University, Xi'an 710004, China

${ }^{4}$ Department of Pharmacology, the Second Affiliated Hospital of Xi'an Jiaotong University, Xi'an 710004, China

Full list of author information is available at the end of the article
}

novel anti-cancer pharmaceutical products must be developed; these products may be obtained from Chinese herbal medicines $[4,5]$. Several herbs have been found to have anti-tumor activity and become the main sources of anti-cancer drugs [6].

Angiogenesis is the formation of new blood vessels from existing vasculature. This process has been associated with the growth and dissemination of solid tumors [7]. The complex process of tumor angiogenesis is associated with basal membrane injury, endothelial cell proliferation, cell migration, and the activation of angiogenic factors, among others [8]. The expressions of several cytokines, such the vascular endothelial growth factor (VEGF) and angiopoietin (Ang), is involved in this formation process [9-11]. Cytokines are important regulators of angiogenesis [12]. Therefore, natural herbs have been considered as potential novel sources of compounds to inhibit vascular [13]. 
Scutellaria barbata D. Don (S. barbata) is a herb used in traditional Chinese medicine, which is widely distributed in certain areas of China and Korea. S. barbata has known anti-inflammatory and anti-tumor effects. Thus, this herb has been clinically used for treating inflammatory diseases and cancer. The crude extracts of $S$. barbata have demonstrated inhibitory effects on numerous human cancers in vitro, including hepatoma, colon cancer, lung cancer, and breast cancer [14-18]. Our previous study confirmed that the extract of $S$. barbata is a potent inhibitor in hepatoma in vitro and in vivo [19]. To date, several flavonoids, alkaloids, polysaccharides, and steroids from $S$. barbata have been characterized [20-24]. In the present study, we investigated the anti-angiogenic effects of total flavonoids of $S$. barbata (TF-SB) in human umbilical vein endothelial cells (HUVECs) and the human HCC cell line MHCC97-H.

\section{Methods}

Reagents

Fetal bovine serum (Gibco BRL, Rockville, MD, USA); DMEM medium (Gibco, USA); 3-(4,5-Dimethylthiazol2-yl)-2,5-diphenyl tetrazolium bromide (MTT) was purchased from Sigma-Aldrich (St. Louis, MO,USA); Matrigel (BD Biosciences, San Jose, CA, USA); human VEGF ELISA kit was purchased from WuHan Boshide Biotechnology Co, Ltd. (WuHan, China).

\section{Preparation of TF-SB from Scutellaria barbata D. Don}

Dried plant materials of $S$. barbata were purchased from Yi Shan Tang Chinese Herbal medicine store (Xi'an, China) and authenticated according to the descriptions found in the Chinese Pharmacopoeia. The original herb was identified as Scutellaria barbata D. Don (SB) by Run-Xia Liu at Medical School of Xi'an Jiaotong University (Xi'an, China). The voucher samples, ZLK-ZY-05 (S. barbata) was deposited at the department of oncology, the Second Affiliated Hospital of Xi'an Jiaotong University.

The material was coarsely ground before extraction. A total of $300 \mathrm{~g}$ of the material was extracted twice with $95 \%$ ethanol for $3 \mathrm{~h}$ in $50^{\circ} \mathrm{C}$. The infusion was filtered through a 1-mm pore-size filter. The leftover on the filter was collected after evaporated. The crude extract was isolated by AB- 8 macroporous adsorption resin column in which $70 \%$ aqueous ethanol was used to elute flavonoids. After treatment with AB-8 resin, the flavonoids purity increased with a recovery of $69 \%$. The total flavonoids were stored at $4^{\circ} \mathrm{C}$ for use.

\section{Cell line and cell culture}

MHCC97-H cells and HUVECs were purchased from the Liver Cancer Institute of Fudan University (Shanghai, China). The cells were grown in DMEM maximal medium containing $10 \%$ inactived fetal bovine serum. Both cell lines were cultured at $37^{\circ} \mathrm{C}$ in $5 \% \mathrm{CO}_{2}$ under humidified environment.

\section{MTT assay for the cell viability of HUVEC cells}

Viability of HUVECs was assessed by the MTT assay. Cells were seeded into 96-well plates at the density of $1 \times 10^{4}$ cells/well. After $12 \mathrm{~h}$, the cells were treated with TF-SB in different concentrations $(0,20,40,80$ and $160 \mu \mathrm{g} / \mathrm{mL}$ ) for $48 \mathrm{~h}$ or $72 \mathrm{~h}$, respectively. MTT were applied to each well after treatment. The supernatant were removed after $4 \mathrm{~h}$ incubation. Subsequently, DMSO were added to each. The supernatants were removed carefully and $150 \mu \mathrm{L}$ of dimethyl sulfoxide (DMSO) were added to each well. The absorbance was measured at $490 \mathrm{~nm}$ through an Enzyme-labeling instrument (ELX800, Bio-Tek, Winooski, VT, USA). This assay was performed in triplicate. The results represented the average value of aborbance from three independent experiments done over multiple days.

\section{In vitro scratch assay}

We used the in vitro scratch assay to assess the activity of TF-SB on migration of HUVECs [25]. HUVECs were seeded in 12-well plates $\left(2 \times 10^{5} /\right.$ well $)$ with complete medium overnight to obtain a full confluent monolayer. After $24 \mathrm{~h}$, the cells were scraped away vertically $24 \mathrm{~h}$ later by pipette tip. Each well was washed twice with PBS to remove debris, and then further incubated for $24 \mathrm{~h}$ in serum-free DMEM medium with different concentrations of TF-SB $(0,40,80$ and $120 \mu \mathrm{g} / \mathrm{mL})$. The distances between the 2 edges of the scratch were photographed on each well using inverted microscope at a magnification of $100 \times$ and analyzed quantitatively.

\section{Tube formation assay}

The ability of HUVECs forming network structures was tested by tube formation assay. As previously described [26], 96-well plates were plated with $50 \mu \mathrm{L}$ matrigel and allowed to polymerize at $37^{\circ} \mathrm{C}$ for $30 \mathrm{~min}$. HUVECs were subsequently seeded on the matrigel followed by addition of different concentrations of TF-SB $(0,40,80$ and $120 \mu \mathrm{g} / \mathrm{mL}$ ) and incubation for $9 \mathrm{~h}$ at $37^{\circ} \mathrm{C}$. The tube-like structures were photographed on each well using a phase-contrast microscope (Olympus, Tokyo, Japan) at a magnification of $100 \times$. To quantify the results, we counted the number of branch points, in which at least 3 tubes joined.

\section{Chick chorioallantoic membrane (CAM) assay}

The CAM assay was performed as previously described [25]. 60 fertilized chicken eggs $(14 \pm 2 \mathrm{~d})$ were purchased from Huxian chicken farm (Xi'an, China). The animals were housed and handled in strict accordance with the 
guidelines of the institutional and national Committees of Animal Use and Protection. The protocol was approved by the Committee on the Ethics of Animal Experiments of Xian Jiaotong University College of Medicine (Certificate No. 22-9601018). All efforts were made to minimize animals' suffering and to keep the numbers of animals used to a minimum.

Briefly, the eggs were incubated at $37^{\circ} \mathrm{C}$ in $40-60 \%$ humidity for $96 \mathrm{~h}$. And then, the eggs were randomly divided into four groups that were treated with different concentrations of TF-SB. After 7 days, a window $(1 \times$ $1.5 \mathrm{~cm}^{2}$ ) was opened in the shell to expose a part of the CAM. Different concentrations of TF-SB samples in $20 \mu \mathrm{L}$ PBS was loaded onto sterilized gelatin sponges $\left(2 \mathrm{~mm}^{2}\right)$ that was then applied to the CAM. After $48 \mathrm{~h}$ of incubation, the neovascular numbers in the CAM around the sponges were photographed with an anatomical microscope (YZ20T4 type). The CAM of the sponge around were observed after hematoxylin and eosin (HE) staining [26]. The relationship between leukocyte infiltration and angiogenesis were analyzed quantitatively.

\section{Measurement of VEGF levels by ELISA}

We used ELISA assay to measure the variation of VEGF levels in MHCC97H cells and HUVECs. The supernatant was collected from different treatment groups. The VEGF level in MHCC97-H cells and HUVECs were measured by ELISA kit (Boshide) according to the manufacturer's instructions. The each well was plated with $0.1 \mathrm{~mL}$ diluted samples in samples buffer and incubated $90 \mathrm{~min}$ at $37^{\circ} \mathrm{C}$. Next, $100 \mu \mathrm{L}$ anti-human VEGF antibody was added and incubated for another $60 \mathrm{~min}$. After washing with PBS for three times, $90 \mu \mathrm{L}$ TMB color liquid was added in the dark for $30 \mathrm{~min}$. And then, the absorbance was measured at $450 \mathrm{~nm}$ after TMB Stop
Solution was applied. All measurements were performed for three times. The data represented average of absorbance value from three independent experiments.

\section{Statistical analysis}

Data were presented as Mean \pm standard deviation (SD). Statistical analysis of the data were performed with Student's $t$-test,one-way analysis of variance (ANOVA) test and linear regression analysis using the Statistical Package for Social Sciences version 13.0 (SPSS Inc, Chicago, IL). $p$ value $<0.05$ was considered statistically significant.

\section{Results}

Identification of TF-SB by high performance liquid chromatography (HPLC)

The components of TF-SB were identified using HPLC. As shown in Figure 1, the main peak was identified as scutellarin (A). Other identified flavonoids in TF-SB included apigenin (B), baicalein (C), luteolin (D). The contents of flavonoids A-D were $67.2 \%, 8.7 \%, 4.6 \%$ and $4.3 \%$, respectively.

\section{Effects of TF-SB on the cell viability of HUVECs}

The MTT assays showed that the cell viabilities of the TF-SB treated groups $(20,40,80$ and $160 \mu \mathrm{g} / \mathrm{mL})$ were suppressed by $24.3 \% \pm 0.1 \%, 30.9 \% \pm 1.5 \%, 55.4 \% \pm 0.9 \%$ and $73.2 \% \pm 0.6 \%$ respectively, after $48 \mathrm{~h}$ treatment (Figure 2). The inhibition rate in the TF-SB treated groups was further decreased after treatment for $72 \mathrm{~h}$, while the inhibitory rate of the $160 \mu \mathrm{g} / \mathrm{mL}$ group reached $78.1 \% \pm 0.6 \%$. Therefore, the anti-proliferative effects of TF-SB on HUVECs occured in a time- and dose-dependent manner.

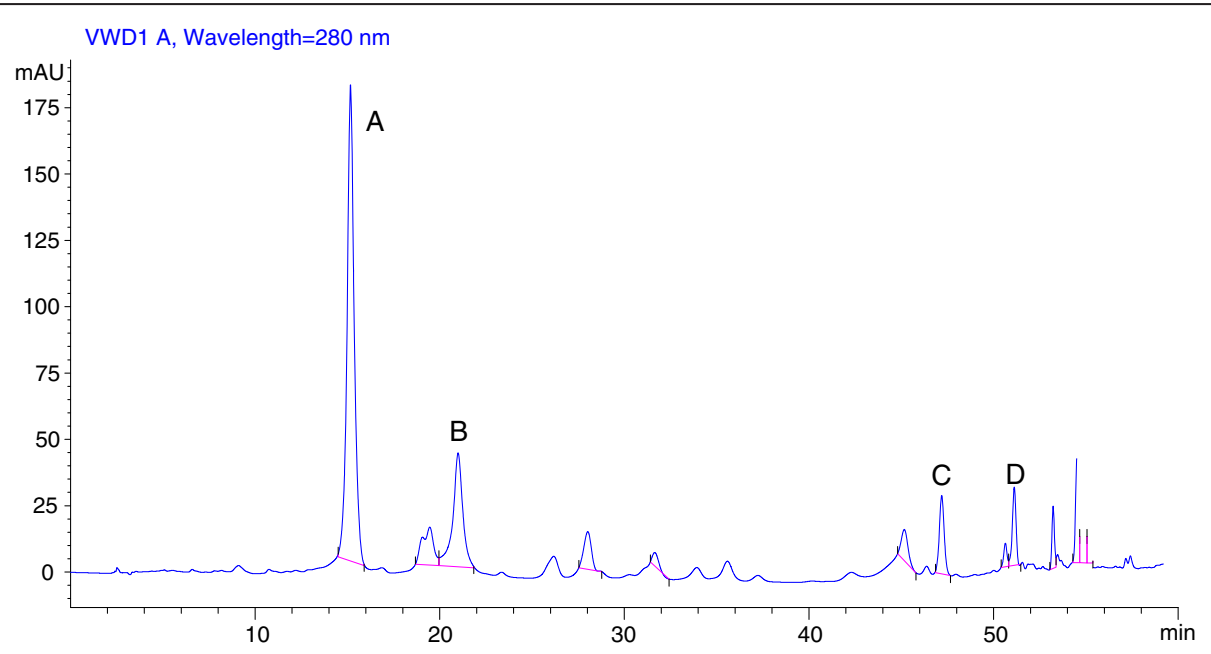

Figure 1 HPLC analysis of TF-SB. There was a main peak in HPLC, which was identified as scutellarin (A). There were also some other flavonoids in TF-SB which were identified as apigenin (B), baicalein (C), luteolin (D). (Wavelength $=280 \mathrm{~nm}$ ). 


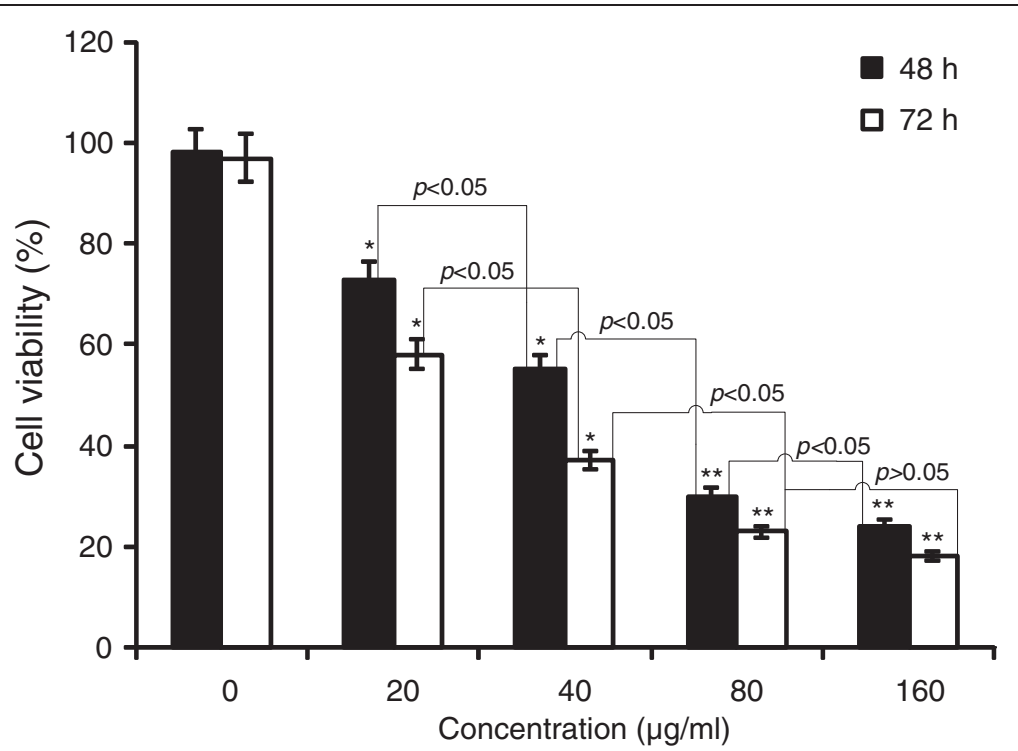

Figure 2 Growth inhibiting effects of TF-SB on HUVECs. Cell viability was determined by MTT method and treated with different concentrations drug for 48 or $72 \mathrm{~h}$. This assay was performed in triplicate $\left(p<0.05\right.$, ANOVA analysis). ${ }^{*} p<0.05,{ }^{* *} p<0.01$ versus the control group.

\section{Effect of TF-SB on the migration of HUVECS}

Endothelial cell migration is a necessary step of angiogenesis [9]. In the present study, the effects of TF-SB on HUVECs migration were determined using the scratch assay. As shown in Figure 3, cell migration in TF-SB groups was inhibited in various degrees after $48 \mathrm{~h}$ of treatment. The maximum inhibition was achieved by the group that received $120 \mu \mathrm{g} / \mathrm{mL}$ TF-SB, which was higher than control group $(p<0.05)$ or those with $40 \mu \mathrm{g} / \mathrm{mL}$ treatment $(p<0.05)$. The cell migration of HUVECs was inhibited in a dose-dependent manner by treatment with TF-SB for $48 \mathrm{~h}$.

\section{TF-SB inhibits HUVEC neovascularization}

The formation of tube-like structures is an essential step in angiogenesis. This process involves matrix degradation, rearrangement and apoptosis of endothelial cells [27]. Therefore, HUVECs angiogenesis was observed using the tube formation assay. As shown in Figure 4, the capillary tube structures were observed in the basal membrane of the control group after HUVECs were placed in the wells. By contrast, the TF-SB treatment significantly reduced the formation of tube-like structures in a dose-dependent manner. Only a few tube-like structures were formed in the $120 \mu \mathrm{g} / \mathrm{mL}$ TF-SB treated group.

\section{Effect of TF-SB on angiogenesis in vivo}

The chicken chorioallantoic membrane (CAM) assay has been widely used for developmental and postdevelopmental studies of angiogenesis because of the easy access to the vascularized CAM $[28,29]$. Moreover, animal experimentation licenses are not compulsory for chicken embryo experiments in many countries. Furthermore, the CAM assay is rapid, inexpensive, and suitable for the large-scale screening of potential angiogenesis regulators [30].

In this study, we used a CAM model to confirm the effect of TF-SB on angiogenesis in vivo. After treatment for $48 \mathrm{~h}$, the blood vessel structures were observed using an anatomical microscope. As shown in Figure 5, a normal vascular pattern with numerous branching was observed in the control group. The total number of blood vessels in the TF-SB treatment was significantly decreased, as compared with the control group $(p<0.05)$. The results indicated that TF-SB could suppress angiogenesis in vivo.

\section{Relationship between the leukocyte infiltration count and blood vessel formation}

The infiltration of macrophages, lymphocytes, and mast cells often presented in tumors microenvironment because these cells may contribute to tumor progression. Previous studies have suggested that these inflammatory cells promote the neoplastic progression by stimulating tumor revascularization and other processes related to tumor angiogenesis [31]. In the present study, the leukocyte infiltration count for the surrounding sponge angiogenesis was observed on the CAM by hematoxylin and eosin (HE) staining. As shown in Figure 6, leukocyte infiltration was not correlated with the formation of large blood vessels. However, the infiltration was positively correlated with the formation of small blood vessels. 


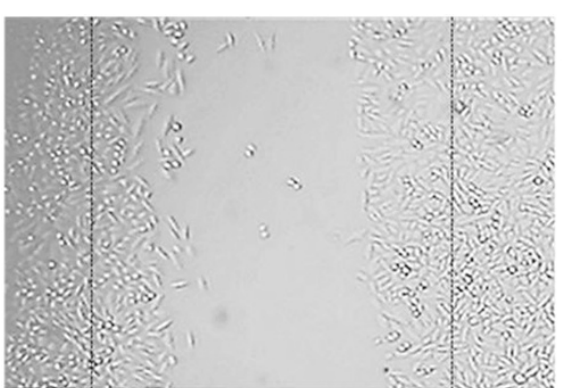

A
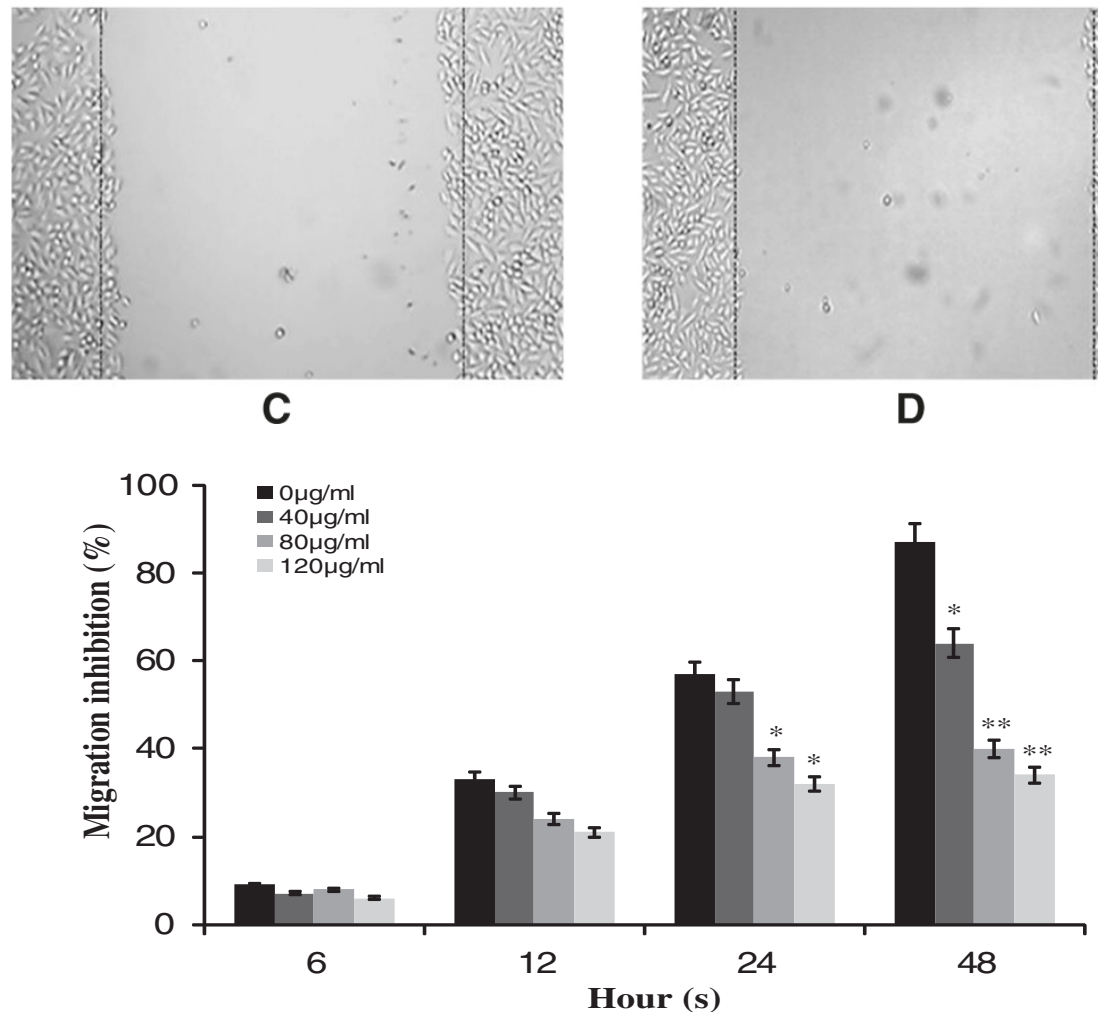

B

D

Figure 3 Effects of TF-SB on the cell migration of HUVECs. Cell migration was analyzed by the scratch assay. HUVECs were treated with various concentrations of TF-SB (40, 80 and $120 \mu \mathrm{g} / \mathrm{mL}$ ) for 48 h. A: blank control group; B: $40 \mu \mathrm{g} / \mathrm{mL}$ TF-SB group; C: $80 \mu \mathrm{g} / \mathrm{mL}$ TF-SB group; D: $120 \mu \mathrm{g} / \mathrm{mL}$ TF-SB group. The images were captured under a phase-contrast microscope at a magnification of 100x. Values represent mean \pm SD from three independent experiments. ${ }^{*} p<0.05,{ }^{* *} p<0.01$ versus the control group.

\section{TF-SB suppresses the expression of VEGF in both MHCC97-H cells and HUVECs}

VEGF is a potent mitogen responsible for the induction of angiogenesis [32]. The humanized monoclonal antibody of VEGF, named bevacizumab, has been used to treating several cancers [33]. In this study, variations of the VEGF levels were measured using ELISA. The cells were treated with different concentrations of TF-SB for 24 or $48 \mathrm{~h}$. The VEGF was then detected in the supernatant culture media. As shown in Figure 7, VEGF expression levels were clearlyly decreased after TF-SB treatment in MHCC97-H cells and HUVECs. Furthermore, the results showed that the VEGF expression
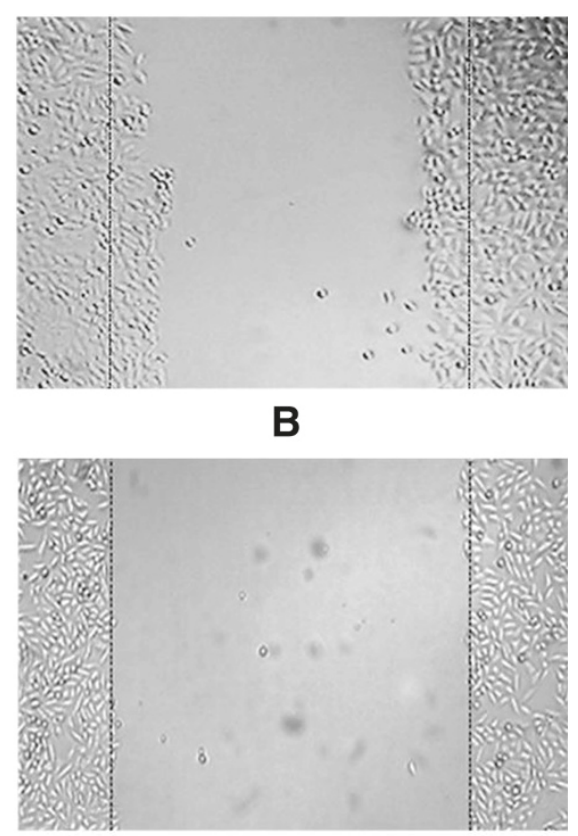

levels were correlated with the various concentrations of TF-SB $(p<0.05)$.

\section{Discussion}

Novel treatments, such as targeted therapy and gene therapy have become an important means of comprehensive cancer treatment for various tumors. However, these therapies have not been widely used because of their limited efficiency and high cost. Traditional Chinese medicine has been used in anti-tumor treatment for thousands of years. These medicines have been used to enhance immune function, and reduce side effects, as well as to prevent recurrence and metastasis for cancer 


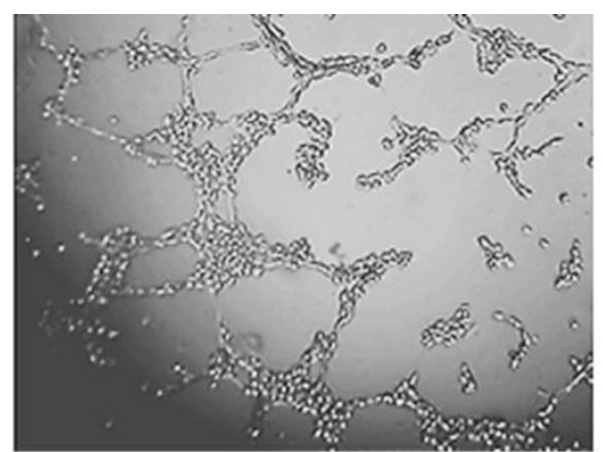

A

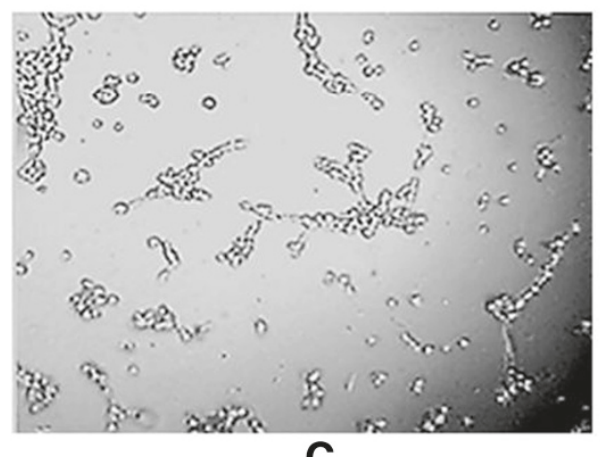

C

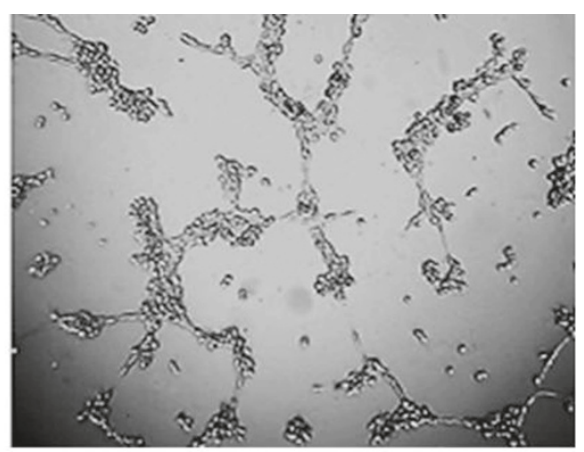

B

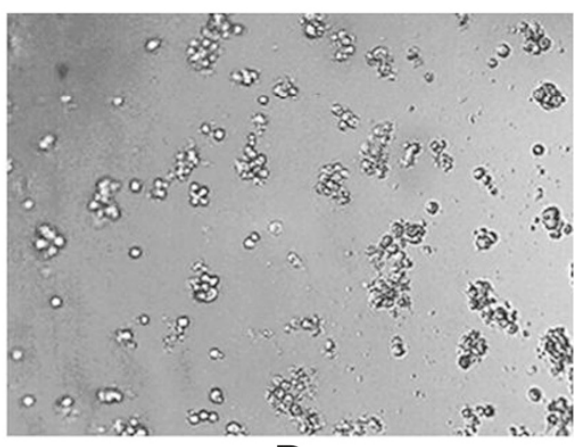

D

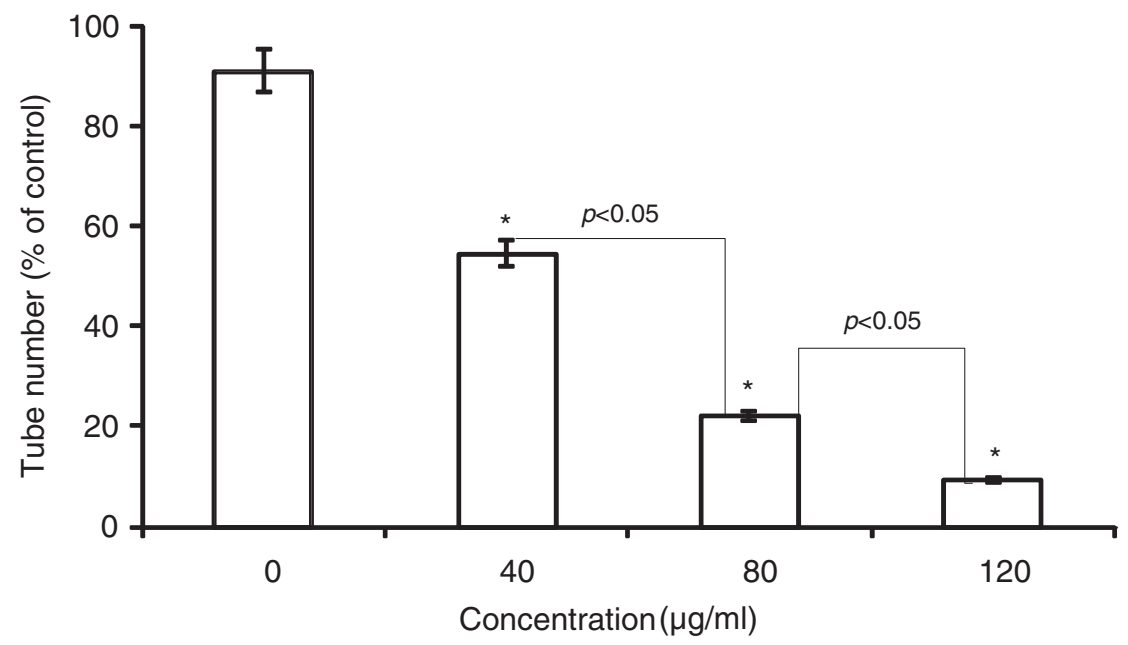

Figure 4 The effect of TF-SB on HUVEC tube formation. HUVECS were seeded on Matrigel-coated 96-well plates and incubated in the diluted medium containing different concentrations of TF-SB for 9 h at $37^{\circ} \mathrm{C}$. A: blank control group; B: $40 \mu \mathrm{g} / \mathrm{mL}$ TF-SB group; C: $80 \mu \mathrm{g} / \mathrm{mL}$ TF-SB group; D: $120 \mu \mathrm{g} / \mathrm{mL}$ TF-SB group. The images were captured under a phase-contrast microscope at a magnification of $100 \times$ and observed the networklike structures. Values represent mean \pm SD from three independent experiments. ${ }^{*} p<0.05,{ }^{* *} p<0.01$ compared with the control group.

patients. The anticancer mechanism and activity of extracts from Chinese herbs have been reported in vitro. For example, BZL101 is an aqueous extract of $S$. barbata with anticancer properties against several human cancers [34]. The crude extract of $S$. barbata similarly has anticancer and anti-angiogenic activity in vitro and in vivo $[19,35]$.

S. barbata is one of the conventional anticancer drugs in China because of its significant anti-tumor activity and inhibition of angiogenesis [36]. S. barbata is effective against a wide range of tumors. The extracts of $S$. barbata greatly could greatly inhibit the cell growth in lung cancer, leukemia, colon cancer, hepatoma, and skin cancer [15-18]. Lee et al. reported that S. barbata decreased the proliferation of myometrial and leiomyomal cells originally promoted by HCG [37]. The chemical composition of $S$. barbata includes flavonoids, diterpenoids, and polysaccharides. Flavonoids are considered the main anti-tumor component of S. barbata. In our study, 


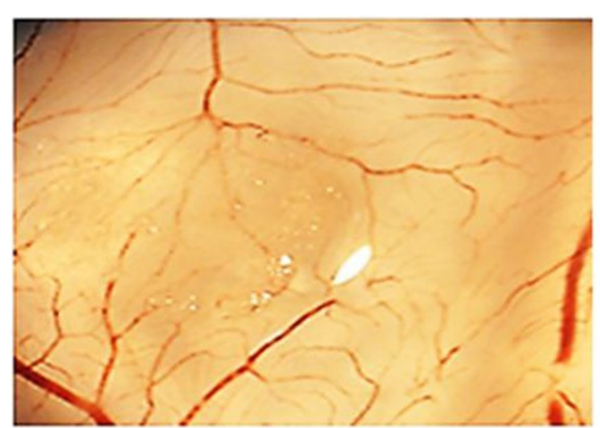

A

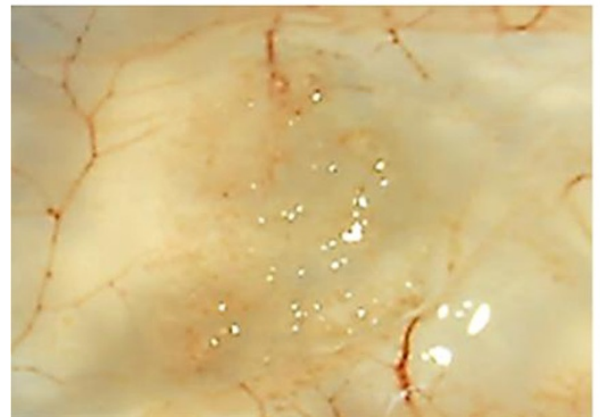

C

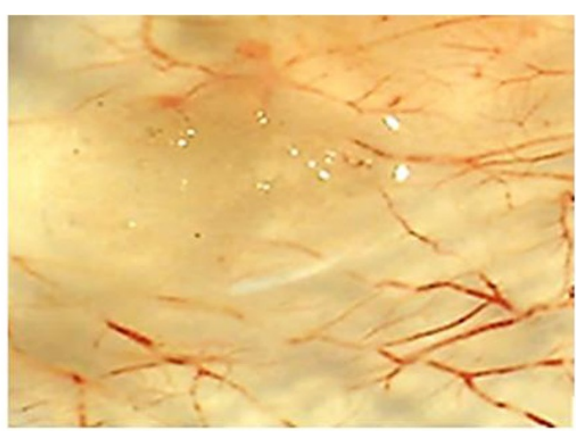

B

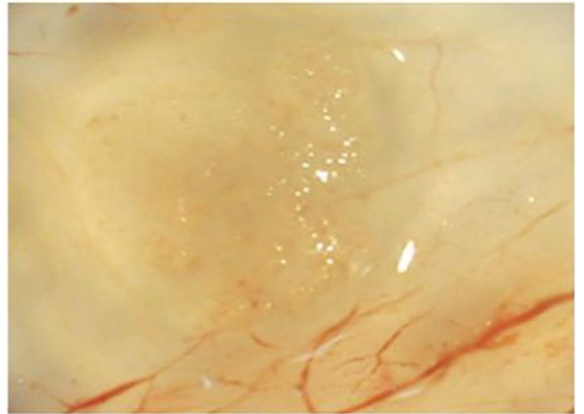

D

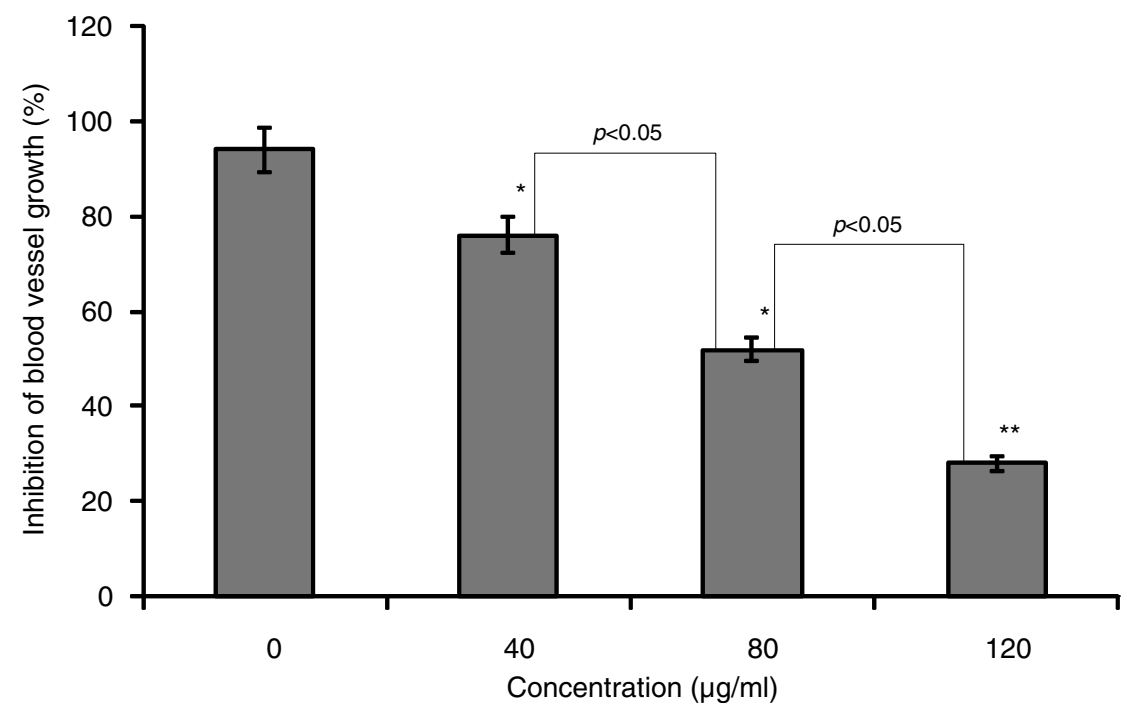

Figure 5 The effects of TF-SB on the angiogenesis of CAM. The chick chorioallantoic membrane of 7-day-old chick embryos were treated with various concentrations of TF-SB and incubated for 48 h. A: blank control group; B: $40 \mu \mathrm{g} / \mathrm{mL}$ TF-SB group; C: $80 \mu \mathrm{g} / \mathrm{mL}$ TF-SB group; D: $120 \mu \mathrm{g} / \mathrm{mL}$ TF-SB group. The angiogenesis around the gelatin sponges was photographed with an anatomical microscope. Values represent mean \pm SD from fifteen eggs. ${ }^{*} p<0.05,{ }^{* *} p<0.01$ compared with the control group.

we found that TF-SB could inhibit angiogenesis based on the proliferation, migration and tube formation of endothelial cells. Our results showed that TF-SB inhibited the proliferation and migration of HUVECs in a dosedependent manner. Thus, TF-SB could significantly suppress the progress of angiogenesis in HUVECs.

Tumor neovascularization is defined as the process of new blood vessel formation in solid neoplasms [27]. The activation of angiogenic pathways is required for tumor spreading, and the proliferation of metastatic cells in distant organs [38]. In a phase IB, multicenter clinical trial of the drug Bezielle in the USA, an aqueous extract of $S$. barbata, was safely administered to the patients and demonstrated promising clinical evidence of anticancer activity in a heavily pretreated population of women with metastatic breast cancer [39]. The ethanol 


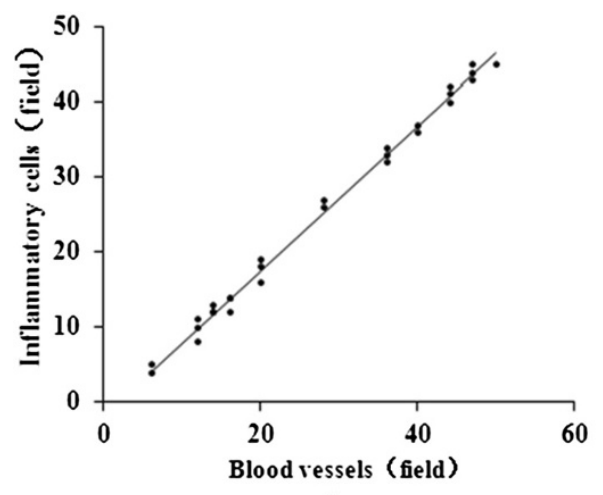

A

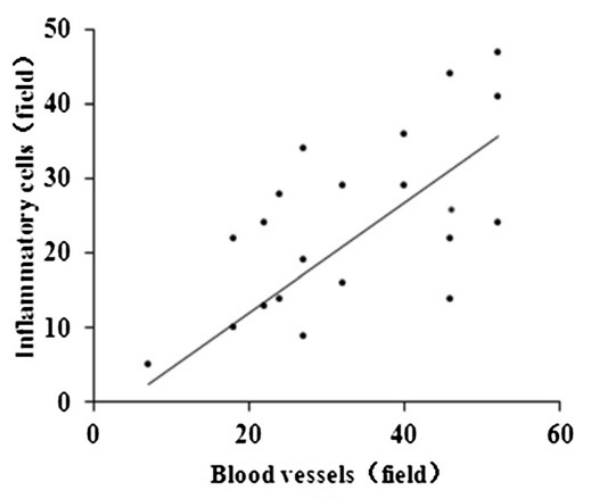

B

Figure $\mathbf{6}$ The relationship between leukocyte infiltration count and formation of blood vessels. The leukocyte infiltration and blood vessels on the CAM were observed under the microscope by HE staining. and no correlation between the formations of big blood vessels. $\mathbf{A}$ : The leukocyte infiltration was positively correlated with small blood vessels $(r=0.883, p<0.05)$; B: The leukocyte infiltration had no correlation with formation of big blood vessels ( $r=0.067, p>0.05$ ).

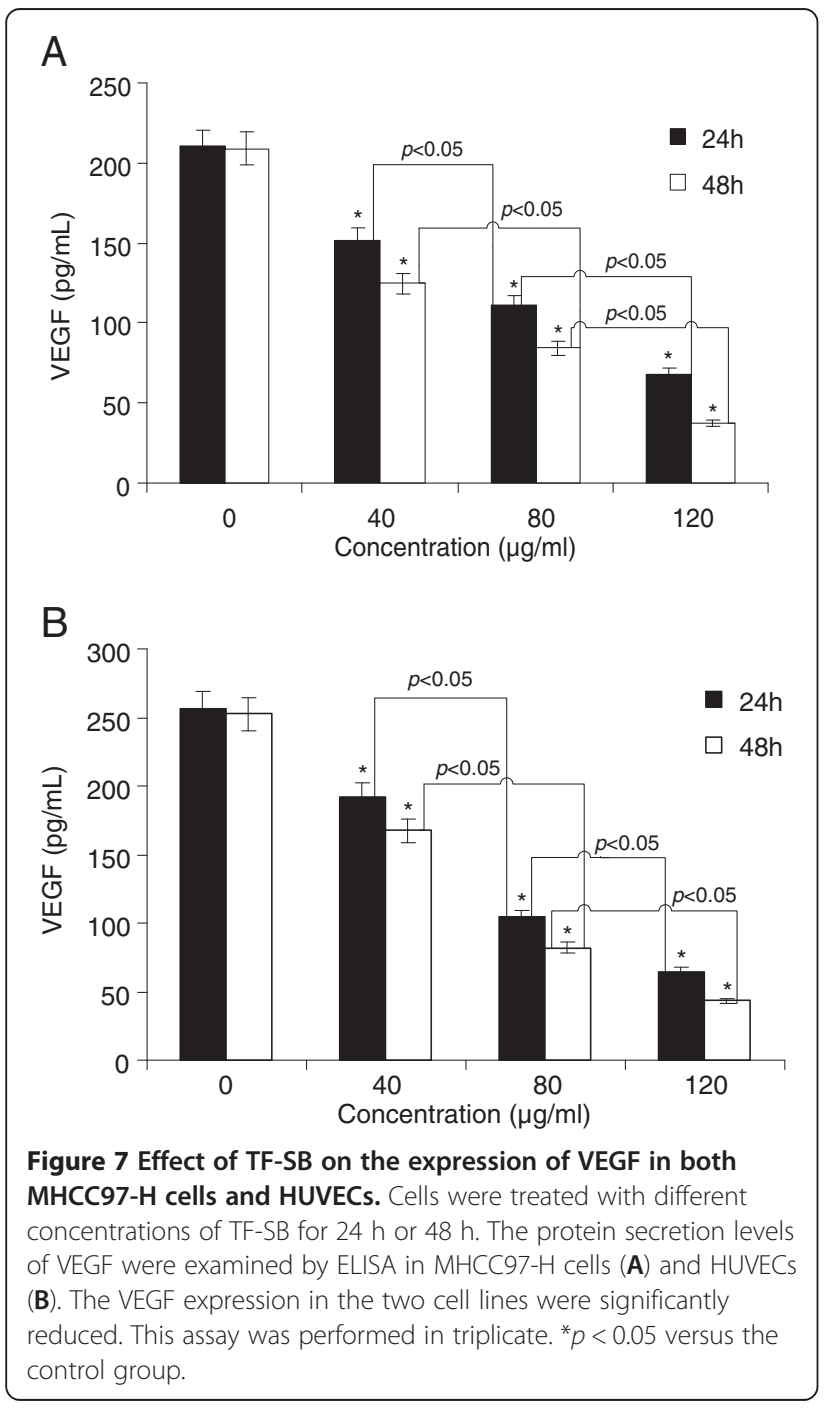

extract of S. barbata could inhibit tumor angiogenesis in a colorectal cancer mouse xenograft model by suppressing the SHH pathway [40]. In the present study, angiogenesis of HUVECs was observed through tube formation assay. We found that TF-SB could significantly suppress the process of angiogenesis of HUVECs.

Inflammation, cytokine activation, and angiogenesis are common features of the tumor microenvironment during the progression of malignancy [41]. These biological processes often share mutual pathways related to cancer progression [42]. Inflammatory cells are macrophages or monocytes induced by various cytokines to become tumor-associated macrophages (TAM) during tumorigenesis. These cells are considered contributing factors for creating the required microenvironment for angiogenesis [43]. In the present study, we found that TF-SB inhibited the angiogenesis of CAM in vivo. Simultaneously, the surrounding sponge layer had numerous infiltrating inflammatory cells by HE staining. A positive correlation was found between leukocyte infiltration and the abundance of small blood vessels.

VEGF may be combined with specific endothelial cell receptors, which are secreted by the autocrine and paracrine pathways to promote angiogenesis [44]. In the present study, the results showed that TF-SB downregulated the expression of VEGF in the MHCC97-H cells and HUVECs.

\section{Conclusion}

In conclusion, TF-SB treatment could significantly suppress the process of angiogenesis in HUVECs grown on matrigels in vitro, and the angiogenesis of CAM in vivo. However, TF-SB is composed of several compounds, including scutellarin, apigenin, baicalein and luteolin, among others. The anticancer effects of these individual components or of various combined ingredients remain unknown. Further experimental studies are required to 
clarify the anticancer molecular mechanisms of TF-SB treatment.

\author{
Abbreviations \\ HCC: Hepatocellular carcinoma; VEGF: Vascular endothelial growth factor; \\ HUVECs: Human umbilical vein endothelial cells; MTT: 3-(4,5-Dimethylthiazol- \\ 2-yl)-2,5-diphenyl tetrazolium bromide; CAM: Chick embryo chorioallantoic \\ membrane; TF-SB: Total flavones of Scutellaria barbatae; HPLC: High \\ performance liquid chromatography.
}

\section{Competing interests}

The authors declare that they have no competing interests.

\section{Authors' contributions}

DZJ, WXJ and WWY designed the research. DZJ, LWF, GJ, KHF and MYG performed the experiments throughout this research. ZSQ, DY and LS contributed to the reagents, and participated in its design and coordination. DZJ and GJ analyzed the data; DZJ and LWF contributed to the writing of the manuscript. Co-first authors: DZJ, LWF and GJ. All authors have read and approved the final manuscript.

\section{Acknowledgments}

This study was supported by National Natural Science Foundation of China, No. 81102711, No. 81274136; the Fundamental Research Funds for the Central Universities, China, No. xjj2011039; Sci-tech Program of Administration of Traditional Chinese Medicine of Shaanxi Province, China.

\section{Author details}

'Department of Oncology, the Second Affiliated Hospital of Xi'an Jiaotong University, Xi'an 710004, China. ${ }^{2}$ Department of Surgical Oncology, Shangluo Central Hospital, Shangluo 726000, China. ${ }^{3}$ Department of Nephrology, the Second Affiliated Hospital of Xi'an Jiaotong University, 710004, Xi'an, China. ${ }^{4}$ Department of Pharmacology, the Second Affiliated Hospital of Xi'an Jiaotong University, Xi'an 710004, China.

Received: 11 January 2013 Accepted: 27 June 2013

Published: 1 July 2013

\section{References}

1. Jemal A, Bray F, Center MM, Ferlay J, Ward E, Forman D: Global cancer statistics. CA Cancer J Clin 2011, 61:69-90

2. Kim DY, Han KH: How to improve treatment outcomes for hepatocellular carcinoma of intermediate and advanced stage. Dig Dis 2012, 30:598-602.

3. Chang CY, Huang ZN, Yu HH, Chang LH, Li SL, Chen YP, Lee KY, Chuu JJ: The adjuvant effects of Antrodia Camphorata extracts combined with anti-tumor agents on multidrug resistant human hepatoma cells. J Ethnopharmacol 2008, 118:387-395.

4. Xu W, Towers AD, Li P, Collet JP: Traditional Chinese medicine in cancer care: perspectives and experiences of patients and professionals in China. Eur J Cancer Care 2006, 15:397-403.

5. Normile D: The new face of traditional Chinese medicine. Science 2003, 299:188-190.

6. Parekh HS, Liu G, Wei MQ: A new dawn for the use of traditional Chinese medicine in cancer therapy. Mol Cancer 2009, 8:21.

7. Mineo M, Garfield SH, Taverna S, Flugy A, DeLeo G, Alessandro R, Kohn EC: Exosomes released by $\mathrm{K} 562$ chronic myeloid leukemia cells promote angiogenesis in a Src-dependent fashion. Angiogenesis 2012, 15:33-45.

8. Nishida N, Yano H, Nishida T, Kamura T, Kojiro M: Angiogenesis in cancer. Vasc Health Risk Manag 2006, 2:213-219.

9. Carmeliet P, Jain RK: Angiogenesis in cancer and other diseases. Nature 2000, 407:249-257.

10. Belting $\mathrm{M}$, Ahamed J, Ruf $\mathrm{W}$ : Signaling of the tissue factor coagulation pathway in angiogenesis and cancer. Arteriosd Throm Vas 2005, 25:1545-1550.

11. Wojtukiewicz MZ, Sierko E, Rak J: Contribution of the hemostatic system to angiogenesis in cancer. Semin Thromb Hemost 2004, 30:5-20.

12. Li JL, Sainson RCA, Shi W, Leek R, Harrington LS, Preusser M, Biswas S, Turley H, Heikamp E, Hainfellner JA, Harris AL: Delta-like 4 notch ligand regulates tumor angiogenesis, improves tumor vascular function, and promotes tumor growth In vivo. Cancer Res 2007, 67:11244-11253.
13. Roodink I, Leenders WPJ: Targeted therapies of cancer angiogenesis inhibition seems not enough. Cancer Lett 2010, 299:1-10.

14. Kim KW, Jin UH, Kim DI, Lee TK, Kim MS, Oh MJ, Kim MS, Kwon DY, Lee YC, Kim CH: Antiproliferative effect of Scutellaria barbata D. Don. on cultured human uterine leiomyoma cells by down-regulation of the expression of Bcl-2 protein. Phytother Res 2008, 22:583-590.

15. Tang PM, Chan JY, Zhang DM, Au SWN, Fong WP, Kong SK, Tsui SKW, Waye MMY, Mak TCW, Fung KP: Pheophorbide a, an active component in Scutellaria barbata, reverses P-glycoprotein-mediated multidrug resistance on a human hepatoma cell line R-HepG2. Cancer Biol Ther 2007, 6:504-509.

16. Goh D, Lee YH, Ong ES: Inhibitory effects of a chemically standardized extract from Scutellaria barbata in human colon cancer cell lines, LoVo. J Agric Food Chem 2005, 53:8197-8204.

17. Yin X, Zhou J, Jie C, Xing DM, Zhang Y: Anticancer activity and mechanism of Scutellaria barbata extract on human lung cancer cell line A549. Life Sci 2004, 75:2233-2244.

18. Suh SJ, Yoon JW, Lee TK, Jin UH, Kim SL, Kim MS, Kwon DY, Lee YC, Kim CH: Chemoprevention of Scutellaria bardata on human cancer cells and tumorigenesis in skin cancer. Phytother Res 2007, 21:135-141.

19. Dai ZJ, Gao J, Li ZF, Ji ZZ, Kang HF, Guan HT, Diao Y, Wang BF, Wang XJ: In vitro and In vivo antitumor activity of Scutellaria barbate extract on murine liver cancer. Molecules 2011, 16:4389-4400.

20. Yu JQ, Liu HB, Lei JC, Tan WJ, Hu XT, Zou GL: Antitumor activity of chloroform fraction of Scutellaria barbata and its active constituents. Phytother Res 2007, 21:817-822.

21. Qu GW, Yue XD, Li GS, Yu QY, Dai SJ: Two new cytotoxic ent-clerodane diterpenoids from Scutellaria barbata. J Asian Nat Prod Res 2010 12:859-864.

22. Dai SJ, Sun JY, Ren Y, Liu K, Shen L: Bioactive ent-clerodane diterpenoids from Scutellaria barbata. Planta Med 2007, 73:1217-1220.

23. Mi X, Zhu R: Simultaneous determination of 7 active ingredients in Scutellaria barbata D. Don by capillary micellar electrokinetic chromatography. Se Pu 2010, 28:209-214.

24. Dai SJ, Peng WB, Shen L, Zhang DW, Ren Y: New norditerpenoid alkaloids from Scutellaria barbata with cytotoxic activities. Nat Prod Res 2011, 15:1-6.

25. Wang XL, Zhang N, Huo Q, Yang Q: Anti-angiogenic and antitumor activities of Huaier aqueous extract. Oncol Rep 2012, 28:1167-1175.

26. Yeh JC, Cindrova-Davies T, Belleri M, Morbidelli L, Miller N, Cho CW, Chan K, Wang YT, Luo GA, Ziche M, Presta M, Charnock-Jones DS, Fan TP: The natural compound $n$-butylidenephthalide derived from the volatile oil of Radix Angelica sinensis inhibits angiogenesis in vitro and in vivo. Angiogenesis 2011, 14:187-197.

27. Kerbel RS: Tumor angiogenesis. N Engl J Med 2008, 358:2039-2049.

28. Borges J, Tegtmeier FT, Padron NT, Mueller MC, Lang EM, Stark GB: Chorioallantoic membrane angiogenesis model for tissue engineering: a new twist on a classic model. Tissue Eng 2003, 9:441-450.

29. Hagedorn M, Balke M, Schmidt A, Bloch W, Kurz H, Javerzat S, Rousseau B, Wilting J, Bikfalvi A: VEGF coordinates interaction of pericytes and endothelial cells during vasculogen-esis and experimental angiogenesis. Dev Dyn 2004, 230:23-33.

30. Kilarski WW, Petersson L, Fuchs PF, Zielinski MS, Gerwins P: An in vivo neovascularization assay for screening regulators of angiogenesis and assessing their effects on pre-existing vessels. Angiogenesis 2012, 15:643-655.

31. Yu JL, Rak JW: Host microenvironment in breast cancer development Inflammatory and immune cells in tumour angiogenesis and arteriogenesis. Breast Cancer Res 2003, 5:83-88.

32. Ferrara N: Molecular and biological properties of vascular endothelial growth factor. J Mol Med 1999, 77:527-543.

33. Delli-Carpini J, Karam AK, Montgomery L: Vascular endothelial growth factor and its relationship to the prognosis and treatment of breast, ovarian, and cervical cancer. Angiogenesis 2010, 13:43-58.

34. Marconett CN, Morgenstern TJ, San Roman AK, Sundar SN, Singhal AK, Firestone GL: BZL101, a phytochemical extract from the Scutellaria barbata plant, disrupts proliferation of human breast and prostate cancer cells through distinct mechanisms dependent on the cancer cell phenotype. Cancer Bio Ther 2010, 10:397-405.

35. Shiau AL, Shen YT, Hsieh JL, Wu CL, Lee CH: Scutellaria barbata inhibits angiogenesis through downregulation of HIF-1 a in lung tumor. Environ Toxicol 2012. doi:10.1002/tox.21763. 
36. Wei LH, Lin JM, Xu W, Hong ZF, Liu XX, Peng J: Inhibition of tumor angiogenesis by Scutellaria barbata D. Don via suppressing proliferation, migration and tube formation of endothelial cells and downregulation of the expression of VEGF-A in cancer cells. J Med Plants Res 2011, 5:3260-3268.

37. Lee TK, Kim DI, Song YL, Lee YC, Kim HM, Kim CH: Differential inhibition of Scutellaria barbata D. Don (Lamiaceae) on HCG-promoted proliferation of cultured uterine leiomyomal and myometrial smooth muscle cells. Immunopharmacol Immunotoxicol 2004, 26:329-342.

38. Su JL, Yang PC, Shih JY, Yang CY, Wei LH, Hsieh CY, Chou CH, Jeng YM, Wang MY, Chang KJ, Hung MC, Kuo ML: The VEGF-C/FIt-4 axis promotes invasion and metastasis of cancer cells. Cancer Cell 2006, 9:209-223.

39. Perez AT, Arun B, Tripathy D, Tagliaferri MA, Shaw HS, Kimmick GG, Cohen I, Shtivelman E, Caygill KA, Grady D, Schactman M, Shapiro CL: A phase IB dose escalation trial of Scutellaria barbata (BZL101) for patients with metastatic breast cancer. Breast Cancer Res Treat 2010, 120:111-118.

40. Wei LH, Lin JM, Xu W, Cai Q, Shen A, Hong Z, Peng J: Scutellaria barbata D. Don inhibits tumor angiogenesis via suppression of hedgehog pathway in a mouse model of colorectal cancer. Int J Mol Sci 2012, 13:9419-9430.

41. Viana CT, Campos PP, Carvalho LA, Cenedezi JM, Lavall L, Lopes MT, Ferreira MA, Andrade SP: Distinct types of tumors exhibit differential grade of inflammation and angiogenesis in mice. Microvasc Res 2012, 86:44-51.

42. Ono M: Molecular links between tumor angiogenesis and inflammation: inflammatory stimuli of macrophages and cancer cells as targets for therapeutic strategy. Cancer Sci 2008, 99:1501-1506.

43. Mantovani A, Schioppa T, Porta C, Allavena P, Sica A: Role of tumorassociated macrophages in tumor progression and invasion. Cancer Metast Rev 2006, 25:315-322.

44. Soker S, Kaefer M, Johnson M, Klagsbrun M, Atala A, Freeman MR: Vascular endothelial growth factor-mediated autocrine stimulation of prostate tumor cells coincides with progression to a malignant phenotype. Am J Pathol 2001, 159:651-659.

doi:10.1186/1472-6882-13-150

Cite this article as: Dai et al: Anti-angiogenic effect of the total

flavonoids in Scutellaria barbata D. Don. BMC Complementary and Alternative Medicine 2013 13:150.

\section{Submit your next manuscript to BioMed Central and take full advantage of:}

- Convenient online submission

- Thorough peer review

- No space constraints or color figure charges

- Immediate publication on acceptance

- Inclusion in PubMed, CAS, Scopus and Google Scholar

- Research which is freely available for redistribution 